\title{
Caring for adults who engage in nonsuicidal self-injury
}

Kaustubh G. Joshi, MD

$\mathrm{N}$ onsuicidal self-injury (NSSI) is the direct and deliberate destruction of body tissue without intent to die. ${ }^{1}$ Cutting is the most common form of NSSI; other methods include burning, scraping/ scratching skin, interfering with wound healing, hitting, biting, self-poisoning, and purposeful non-recreational risk-taking. ${ }^{2,3}$ Although most individuals who engage in NSSI have no intention to die, suicidal ideation often precedes the initial engagement in NSSI, ${ }^{4}$ and a history of repeated NSSI is a risk factor for suicide attempts. ${ }^{4}$ In a systematic review, Cipriano et $\mathrm{al}^{5}$ found that NSSI is most common among adolescents and young adults, with onset most often occurring between age 12 and 14 . Prevalence rates of NSSI are $7.5 \%$ to $46.5 \%$ in adolescents, $38.9 \%$ in university students, and $4 \%$ to $23 \%$ in adults. ${ }^{5}$

Although no medications have consistently shown efficacy for treating NSSI, research suggests cognitive-behavioral therapy and dialectical behavioral therapy may be helpful. Unfortunately, these therapies are often not available during a patient's acute crisis. ${ }^{3}$ Because a thorough review of the treatment options for NSSI is beyond the scope of this article, here I offer tips for caring for adults who engage in NSSI. Although there are slight differences in managing NSSI in adolescents (eg, the need for parental monitoring and reducing risk of contagion), these tips also can be used with adolescents.

\section{Explore why your patient engages in NSSI.} Identifying the reasons for our patients' NSSI makes it easier for us to empathize with them, and puts us in a better position to treat them. ${ }^{3}$ The most widely reported reasons for
NSSI are to cope with distress/anguish and to exert influence on others. ${ }^{6}$ In a systematic review, self-reported reasons for NSSI also included punishing oneself for having positive feelings, punishing others, managing dissociation (ie, active pursuit of numbness), sensation-seeking (ie, to generate excitement or exhilaration), averting suicide (ie, warding off suicidal thoughts), maintaining or exploring boundaries, and expressing or coping with sexuality. ${ }^{6}$ When exploring your patient's reasons for NSSI, determine if the behavior is based on a true suicidal desire. Because NSSI is associated with mood disorders, anxiety disorders, personality disorders, and other disorders, also assess for any underlying psychiatric conditions, and treat them accordingly because mental health treatment has been empirically proven to reduce suicide rates. ${ }^{2,7}$

\section{Conduct a suicide risk assessment.} Regardless of your patient's reasons for NSSI, an individualized and thorough suicide risk assessment is needed to identify modifiable, non-modifiable, and protective factors that you can consider when developing a treatment plan. Key components of such assessments include (but continued on page 45

\section{Every issue of CURRENT PsychatRY} has its 'Pearls'

\section{Yours could be found here.}

Read the 'Pearls' guidelines for manuscript submission at MDedge.com/ CurrentPsychiatry/page/pearls. Then, share with your peers a 'Pearl' of wisdom from your practice.
Dr. Joshi is Associate Professor of Clinical Psychiatry, and Associate Director, Forensic Psychiatry Fellowship, Department of Neuropsychiatry and Behavioral Science, University of South Carolina School of Medicine, Columbia, South Carolina. He is one of CURRENT Psychiatry's Department Editors for Pearls.

\section{Disclosure}

The author reports no financial relationships with any companies whose products are mentioned in this article, or with manufacturers of competing products.

doi: $10.12788 /$ cp.0081 
Nonsuicidal self-injury

continued from page 11

are not limited to) current and past urges to engage in NSSI, past NSSI and suicide attempts, access to lethal means, and ability to follow a safety plan.

Avoid exaggerating the danger and importance of NSSI. Treating a patient who engages in NSSI who is motivated by a true suicidal desire and/or has underlying psychiatric conditions may prompt you to consider hospitalization and/or prescribing psychotropic medications. ${ }^{3}$ However, because most NSSI is not due to a true suicidal desire, overreacting may unwittingly communicate to the patient that self-harm is a way to sustain someone's attention, thus reinforcing that such behaviors can help them obtain support when distressed. ${ }^{3}$ Further, overreacting will not help patients comprehend and better cope with the reasons for their selfinjurious behaviors. ${ }^{3}$

\section{Restrict your patient's access to lethal}

means. Restricting access to items such as firearms, sharp objects (eg, knives and razors), medications, implements for suffocation/hanging (eg, belts), and household poisons has been empirically proven to reduce suicide rates. ${ }^{7}$ Such restrictions can also potentially reduce the likelihood of NSSI. It is important to repeatedly ask your patient if they have acquired any new means, and to listen for information that indicates they possess means that they did not previously disclose. It is also important to ask if the patient has moved existing means to an area for easier access to use them.

Create a safety plan. Written safety plans can include a list of warning signs (thoughts, images, mood, situations, behaviors) that a crisis is developing, coping strategies (eg, going for a walk, exercising, engaging in a hobby, socializing with friends or family), and contact information for 24-hour crisis hotlines, emergency rooms, and mental health clinicians. ${ }^{8}$ The Suicide Prevention
Resource Center offers a safety plan template at www.sprc.org/sites/default/files/ resource-program/Brown_StanleySafety PlanTemplate.pdf. ${ }^{8}$

Offer empathy. Individuals who engage in NSSI are making a desperate call for help that requires concerned and supportive responses. ${ }^{3}$ One such response is to provide empathy. In addition to expressing concern and compassion, empathy involves recognizing and sharing your patients' emotions. Empathy also can help you avoid any resistance during the visit by considering what is appropriate to say to patients.

Manage countertransference. You may have negative feelings toward a patient who engages in NSSI, or may even view self-harm as a willful act designed to gain attention. However, such feelings could lead you to minimize or dismiss the importance of your patient's behaviors, which may push them to engage in more dangerous self-harm. ${ }^{3}$ Acknowledging any feelings of derision for a patient who engages in NSSI and understanding why you have these emotions will help you better understand your patient, improve rapport, and ensure that you are not impeding the delivery of appropriate clinical care.

\section{References}

1. Nock MK. Self-injury. Annu Rev Clin Psychol. 2010;6: 339-363.

2. Klonsky ED. Non-suicidal self-injury in United States adults: prevalence, sociodemographics, topography and functions. Psychol Med. 2011;41(9):1981-1986.

3. Gunderson JG, Choi-Kain LW. Working with patients who self-injure. JAMA Psychiatry. 2019;76(9):976-977.

4. Glenn CR, Lanzillo EC, Esposito EC, et al. Examining the course of suicidal and nonsuicidal self-injurious thoughts and behaviors in outpatient and inpatient adolescents. J Abnorm Child Psychol. 2017;45(5):971-983.

5. Cipriano A, Cella S, Cotrufo P. Nonsuicidal self-injury: a systematic review. Front Psychol. 2017;8:1946. doi: 10.3389/ fpsyg.2017.01946

6. Edmondson AJ, Brennan CA, House AO. Non-suicidal reasons for self-harm: a systematic review of self-reported accounts. J Affect Disord. 2016;191:109-117.

7. Mann JJ, Apter A, Bertolete J. Suicide prevention strategies: a systematic review. JAMA. 2005;294(16):2064-2074.

8. Stanley B, Brown GK. Safety planning intervention: a brief intervention to mitigate suicide risk. Cog Behav Practice. 2012;19:256-264
Overreacting

to NSSI may communicate that self-harm is a way to sustain someone's attention

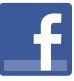

Discuss this article at www.facebook.com/ MDedgePsychiatry (6) 